\title{
Parameter-free ansatz for inferring ground state wave functions of even convex potentials
}

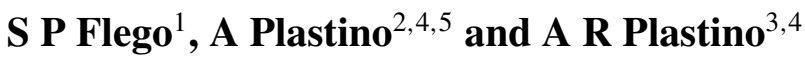 \\ ${ }^{1}$ Universidad Nacional de La Plata, Facultad de Ingeniería, Grupo de Investigación Teórica y Aplicada \\ en Teoría de la Información (GTyATI), 1900 La Plata, Argentina \\ ${ }^{2}$ Universidad Nacional de La Plata, Instituto de Física (IFLP-CCT-CONICET), CC 727, 1900 La Plata, \\ Argentina \\ ${ }^{3}$ CREG-Universidad Nacional de La Plata-CONICET, CC 727, 1900 La Plata, Argentina \\ ${ }^{4}$ Instituto Carlos I de Fisica Teorica y Computacional and Departamento de Fisica Atomica, \\ Molecular y Nuclear, Universidad de Granada, Granada, Spain \\ ${ }^{5}$ Universitat de les Illes Balears and IFISC-CSIC, 07122 Palma de Mallorca, Spain \\ E-mail: angeloplastino@gmail.com
}

Received 21 July 2011

Accepted for publication 13 March 2012

Published 18 April 2012

Online at stacks.iop.org/PhysScr/85/055002

\begin{abstract}
Schrödinger's equation (SE) and the information-optimizing principle based on Fisher's information measure are intimately linked (Frieden et al 1999 Phys. Rev. E 60 48), which entails the existence of a Legendre transform structure underlying the SE (Flego et al $2011 \mathrm{~J}$. Math. Phys. 52 082103). In this paper, we show that the existence of such a structure allows, via the virial theorem, for the formulation of a parameter-free ground state's SE ansatz for a rather large family of potentials. The parameter-free nature of the ansatz derives from the structural information it incorporates through its Legendre properties.
\end{abstract}

PACS numbers: 05.45+b, 05.30-d

(Some figures may appear in colour only in the online journal)

\section{Introduction}

A few quantum-mechanical models admit exact solutions. Approximations of diverse type constitute the hard core of armory at the disposal of the quantum practitioner. Since the 1960s, hypervirial theorems have been gainfully incorporated into the pertinent arsenal $[1,2]$. Here we revisit the subject in an information-theory context, via Fisher's information measure (FIM) with emphasis on (i) its Legendre properties and (ii) its relation to the virial theorem. Why Fisher information? Because

- Schrödinger's equation (SE) and FIM have been shown to be intimately linked (see, e.g., [3-8]). In fact, from these references one infers that FIM works as a kind of 'action' from which a Lagrangian can be built whose variation yields the SE. Moreover,

- FIM can be interpreted as the expectation value of the kinetic term of the SE [9].
Note that the notion of using a small set of relevant expectation values so as to describe the main properties of physical systems may be considered the leitmotiv of statistical mechanics [10]. Developments based on Jaynes' maximum entropy principle constitute a pillar of our present understanding of the discipline [11]. This type of ideas has also been fruitfully invoked to obtain the probability distribution associated with pure quantum states via Shannon's entropy (see, e.g., [12] and references therein). In such a spirit, Fisher information, the local counterpart of the global Shannon quantifier [13], first introduced for statistical estimation purposes [13], has been shown to be quite useful for the variational characterization of quantal equations of motion [14]. In particular, as mentioned above, it is well known that a strong link exists between the FIM $I$ and Schrödinger's wave equation (SE) [3-9]. Such a connection is based on the fact that a constrained Fisher minimization leads to an SE-like equation 
[3-9, 13]. In turn, this guarantees the existence of intriguing relationships between various quantum quantities reminiscent of the ones that characterize thermodynamics due to its Legendre-invariance structure [3, 5, 9]. Interestingly enough, SE consequences such as the Hellmann-Feynman and the virial theorems can be re-interpreted in terms of thermodynamics' Legendre reciprocity relations (RR) [6-8], a fact suggesting that a Legendre-transform structure underlies the non-relativistic SE. As a consequence, the possible energy eigenvalues become constrained by such a structure in a rather unsuspected way [7-9], which allows one to obtain a first-order differential equation, unrelated to SE [9], that energy eigenvalues must necessarily satisfy. The predictive power of that equation was explored in [15], where the formalism was applied to the quantum anharmonic oscillator. Exploring further the interesting properties of this 'quantal-Legendre' structure will occupy us below. We will find, as a consequence of the Legendre symmetry that underlies the Fisher-Schrödinger connection, an elegant expression, in terms of quadratures, for the ground state (GS) wave function associated with a rather large class of potential functions.

\section{Basic ideas}

A special and particularly useful FIM expression (not the most general one) is to be quoted. Let $x$ be a stochastic variable and $f(x)=\psi(x)^{2}$ the probability density function (PDF) for this variable. Then $I$ reads [13]

$$
I=\int f(x)\left[\frac{\partial \ln f(x)}{\partial x}\right]^{2} \mathrm{~d} x=4 \int\left[\frac{\partial \psi(x)}{\partial x}\right]^{2} \mathrm{~d} x, \quad f=\psi^{2}
$$

Let us focus our attention now on a system that is specified by a set of $M$ physical parameters $\mu_{k}$. We can write $\mu_{k}=\left\langle A_{k}\right\rangle$, with $A_{k}=A_{k}(x)$. The set of $\mu_{k}$-values is to be regarded as our prior knowledge (available empirical information). Again, the PDF is called $f(x)$. Then,

$$
\left\langle A_{k}\right\rangle=\int \mathrm{d} x A_{k}(x) f(x), \quad k=1, \ldots, M .
$$

It can be shown (see $[3,4])$ that the physically relevant PDF $f(x)$ minimizes FIM subject to the prior conditions and the normalization condition. Normalization entails $\int \mathrm{d} x f(x)=1$ and, consequently, our Fisher-based extremization problem becomes

$$
\delta\left(I-\alpha \int \mathrm{d} x f(x)-\sum_{k=1}^{M} \lambda_{k} \int \mathrm{d} x A_{k}(x) f(x)\right)=0,
$$

with $(M+1)$ Lagrange multipliers $\lambda_{k}\left(\lambda_{0}=\alpha\right)$. See [3] for details of how to go from (3) to an SE that yields the desired PDF in terms of the amplitude $\psi(x)$. This SE is of the form

$$
\left[-\frac{1}{2} \frac{\partial^{2}}{\partial x^{2}}+U(x)\right] \psi=\frac{\alpha}{8} \psi, \quad U(x)=-\frac{1}{8} \sum_{k=1}^{M} \lambda_{k} A_{k}(x),
$$

and is to be interpreted as the (real) SE for a particle of unit mass $(\hbar=1)$ moving in the effective, 'information-related pseudo-potential' $U(x)$ [3] in which the normalization-Lagrange multiplier $(\alpha / 8)$ plays the role of an energy eigenvalue. The $\lambda_{k}$ are fixed with recourse to the available prior information. For one-dimensional (1D) scenarios, $\psi(x)$ is real [16] and

$$
\begin{aligned}
I & =\int \psi^{2}\left(\frac{\partial \ln \psi^{2}}{\partial x}\right)^{2} \mathrm{~d} x=4 \int\left(\frac{\partial \psi}{\partial x}\right)^{2} \mathrm{~d} x \\
& =-4 \int \psi \frac{\partial^{2}}{\partial x^{2}} \psi \mathrm{d} x
\end{aligned}
$$

so from (4) one finds a simple and convenient $I$ expression

$$
I=\alpha+\sum_{k=1}^{M} \lambda_{k}\left\langle A_{k}\right\rangle
$$

\subsection{The Legendre structure}

The connection between the variational solutions $f$ and thermodynamics was established in [3] and [5] in the guise of typical Legendre RR. These constitute thermodynamics' essential formal ingredient [17] and were re-derived à la Fisher in [3] by recasting (6) in a manner that emphasizes the role of the relevant independent variables,

$$
I\left(\left\langle A_{1}\right\rangle, \ldots,\left\langle A_{M}\right\rangle\right)=\alpha+\sum_{k=1}^{M} \lambda_{k}\left\langle A_{k}\right\rangle
$$

Obviously, the Legendre transform's main goal is to change the identity of our relevant independent variables. As to the normalization multiplier $\alpha$, which plays the role of an energy eigenvalue in equation (4), we have

$$
\alpha\left(\lambda_{1}, \ldots, \lambda_{M}\right)=I-\sum_{k=1}^{M} \lambda_{k}\left\langle A_{k}\right\rangle
$$

After these preliminaries we straightforwardly encounter the three RR [3]

$$
\frac{\partial \alpha}{\partial \lambda_{k}}=-\left\langle A_{k}\right\rangle, \quad \frac{\partial I}{\partial\left\langle A_{k}\right\rangle}=\lambda_{k}, \quad \frac{\partial I}{\partial \lambda_{i}}=\sum_{k}^{M} \lambda_{k} \frac{\partial\left\langle A_{k}\right\rangle}{\partial \lambda_{i}},
$$

the last one being a generalized Fisher-Euler theorem.

\section{The Fisher measure and quantum mechanical connection}

Since the potential function $U(x)$ belongs to $\mathcal{L}_{2}$, it admits a series expansion in the basis $x, x^{2}, x^{3}, \ldots$ [18]. The $A_{k}(x)$ themselves belong to $\mathcal{L}_{2}$ as well and can also be series-expanded in a similar fashion. This enables us to base our future considerations on the assumption that the $a$ priori knowledge refers to moments $x^{k}$ of the independent variable, i.e. $\left\langle A_{k}\right\rangle=\left\langle x^{k}\right\rangle$, and that one possesses information about $M$ of these moments $\left\langle x^{k}\right\rangle$. Our 'information' potential $U$ thus reads

$$
U(x)=-\frac{1}{8} \sum_{k} \lambda_{k} x^{k} .
$$


We will assume that the first $M$ terms of the above series yield a satisfactory representation of $U(x)$. Consequently, the Lagrange multipliers are identified with $U(x)$ 's series-expansion's coefficients.

In a Schrödinger scenario the virial theorem states that [7]

$$
\left\langle\frac{\partial^{2}}{\partial x^{2}}\right\rangle=-\left\langle x \frac{\partial}{\partial x} U(x)\right\rangle=\frac{1}{8} \sum_{k=1}^{M} k \lambda_{k}\left\langle x^{k}\right\rangle
$$

and thus, from (5) and (11), a useful, virial-related expression for FIM can be arrived at [7]:

$$
I=-\sum_{k=1}^{M} \frac{k}{2} \lambda_{k}\left\langle x^{k}\right\rangle
$$

Equation (12) encodes the information provided by the virial theorem $[7,8]$.

Interestingly enough, the RR (9) can be re-derived on a strictly pure quantum mechanical basis [7], starting from the quantum virial theorem (which leads to equation (12)) plus information provided by the quantum Hellmann-Feynman theorem. This fact strongly suggests that a Legendre structure underlies the 1D SE [7]. Thus, with $\left\langle A_{k}\right\rangle=\left\langle x^{k}\right\rangle$, our 'new' $\mathrm{RR}$ are given by

$$
\frac{\partial \alpha}{\partial \lambda_{k}}=-\left\langle x^{k}\right\rangle, \quad \frac{\partial I}{\partial\left\langle x^{k}\right\rangle}=\lambda_{k}, \quad \frac{\partial I}{\partial \lambda_{i}}=\sum_{k}^{M} \lambda_{k} \frac{\partial\left\langle x^{k}\right\rangle}{\partial \lambda_{i}}
$$

and FIM expresses the relation between the independent variables or control variables (the prior information) and $I$. Such information is encoded in the functional form $I=I\left(\left\langle x^{1}\right\rangle, \ldots,\left\langle x^{M}\right\rangle\right)$. For later convenience, we will also denote such a relation or encoding process as $\left\{I,\left\langle x^{k}\right\rangle\right\}$. We see that the Legendre transform FIM structure involves both eigenvalues of the 'information Hamiltonian' and Lagrange multipliers. Information is encoded in $I$ via these Lagrange multipliers, i.e. $\alpha=$ $\alpha\left(\lambda_{1}, \ldots \lambda_{M}\right)$, together with a bijection $\left\{I,\left\langle x^{k}\right\rangle\right\} \leftrightarrow\left\{\alpha, \lambda_{k}\right\}$.

In $a\left\{I,\left\langle x^{k}\right\rangle\right\}$ scenario, the $\lambda_{k}$ are functions dependent on the $\left\langle x^{k}\right\rangle$-values. As shown in [8], substituting the RR given by (13) into (12), one is led to a linear PDE for $I$,

$$
\lambda_{k}=\frac{\partial I}{\partial\left\langle x^{k}\right\rangle} \longrightarrow I=-\sum_{k=1}^{M} \frac{k}{2}\left\langle x^{k}\right\rangle \frac{\partial I}{\partial\left\langle x^{k}\right\rangle}
$$

and a complete solution is given by

$$
I\left(\left\langle x^{1}\right\rangle, \ldots,\left\langle x^{M}\right\rangle\right)=\sum_{k=1}^{M} C_{k}\left|\left\langle x^{k}\right\rangle\right|^{-2 / k},
$$

where $C_{k}$ are positive real numbers (integration constants). The $I$ domain is $D_{I}=\left\{\left(\left\langle x^{1}\right\rangle, \ldots,\left\langle x^{M}\right\rangle\right) /\left\langle x^{k}\right\rangle \in \Re_{o}\right\}$. Equation (15) states that for $\left\langle x^{k}\right\rangle>0, I$ is a monotonically decreasing function of $\left\langle x^{k}\right\rangle$, and as one expects from a 'good' information measure [13], $I$ is a convex function. We may obtain $\lambda_{k}$ from the RR (13). For $\left\langle x^{k}\right\rangle>0$ one gets

$$
\lambda_{k}=\frac{\partial I}{\partial\left\langle x^{k}\right\rangle}=-\frac{2}{k} C_{k}\left\langle x^{k}\right\rangle^{-(2+k) / k}<0
$$

and then, using (6), we obtain the $\alpha$-normalization Lagrange multiplier. For a discussion on how to obtain the reference quantities $C_{k}$, see [15].

The general solution to the I PDE does exist and uniqueness has been demonstrated via an analysis of the associated Cauchy problem [8]. Thus, equation (15) implies what seems to be a kind of 'universal' prescription, a linear PDE that any variationally (with constraints) obtained FIM must necessarily comply with.

\section{The present results}

A good SE ansatz eigenfunction can be formulated via PDFs that satisfy the virial theorem. This constitutes the main result of the present study.

\subsection{Inferring the probability density function for even convex potentials}

The PDF ansatz can be straightforwardly derived from the FIM definition (1) and the virial theorem (11), the procedure being as follows. Begin with the Fisher measure I, 'virially' expressed as

$$
I=-4\left\langle\frac{\partial^{2}}{\partial x^{2}}\right\rangle=4\left\langle x \frac{\partial U}{\partial x}\right\rangle
$$

which, in the Fisher scenario, can obviously be written as

$$
\int \mathrm{d} x f(x)\left(\frac{\partial \ln f(x)}{\partial x}\right)^{2}=4 \int \mathrm{d} x f(x) x \frac{\partial}{\partial x} U(x)
$$

or

$$
\int \mathrm{d} x f(x)\left[\left(\frac{\partial \ln f(x)}{\partial x}\right)^{2}-4 x \frac{\partial}{\partial x} U(x)\right]=0 .
$$

A polynomial is convex iff its Hessian is positive semi-definite. Convex polynomials constitute an important set with many applications [19]. For even convex potentials we have

$$
\forall x, \quad x \frac{\partial}{\partial x} U(x) \geqslant 0
$$

For them we can devise an ansatz $f_{\mathrm{A}}$ that, by construction, verifies (19). We merely require the fulfilment of

$$
\left(\frac{\partial \ln f_{\mathrm{A}}(x)}{\partial x}\right)^{2}-4 x \frac{\partial}{\partial x} U(x)=0 .
$$

Clearly, we immediately obtain

$$
\left(\frac{\partial \ln f_{\mathrm{A}}(x)}{\partial x}\right)^{2}=4 x \frac{\partial}{\partial x} U(x),
$$

which leads to the independent solutions

$$
f_{\mathrm{A}}^{ \pm}(x)=A \exp \left( \pm 2 \int \mathrm{d} x \sqrt{x \frac{\partial}{\partial x} U(x)}\right),
$$

where $A$ is an integration constant. Equation (23) provides the tools for building a nice, rather general and virially motivated 
wave function ansatz. For any even, convex 'information' potential $U(10)$, with $\lambda_{k} \leqslant 0$, we have

$$
f_{\mathrm{A}}^{ \pm}(x)=A \exp \left( \pm \int \mathrm{d} x \sqrt{\sum_{k}-k \lambda_{2 k} x^{2 k}}\right) .
$$

Is the ansatz good enough for our FIM treatment? In the following section, we try to answer such a query.

\section{Ground state eigenfunction for the Schrödinger equation}

We outline here the methodology for constructing the GS ansatz for an SE of the form

$$
\left[-\frac{1}{2} \frac{\mathrm{d}^{2}}{\mathrm{~d} x^{2}}+U(x)\right] \psi(x)=E \psi(x)
$$

where $U(x)$ is a general even-convex potential. According to [6], we can ascribe to (25) a Fisher measure via

$$
f(x)=\psi^{2}(x)
$$

Accordingly, from (23) we obtain

$$
\psi_{\mathrm{A}}^{ \pm}(x)=A \exp \left( \pm \int \mathrm{d} x \sqrt{x \frac{\partial}{\partial x} U(x)}\right),
$$

from which one can construct the SE ansatz eigenfunction.

\subsection{Ground state eigenfunction of an even, convex anharmonic oscillator}

The potential function is given by

$$
U(x)=\sum_{k=1}^{M} a_{2 k} x^{2 k}, \quad a_{2 k} \geqslant 0, \quad k \in \mathcal{N} .
$$

Substituting (28) into (27),

$$
\psi_{\mathrm{A}}^{ \pm}(x)=A \exp \left( \pm \int \mathrm{d} x \sqrt{\sum_{k=1}^{M} 2 k a_{2 k} x^{2 k}}\right)
$$

from which, using appropriate boundary conditions, we can select the appropriate solution and obtain our SE ansatz eigenfunction. As an illustration of the procedure, below we deal with (i) the harmonic oscillator and (ii) the quartic anharmonic oscillator.

\subsection{Harmonic oscillator}

The SE for a particle of unit mass in a harmonic potential reads

$$
\left[-\frac{1}{2} \frac{\partial^{2}}{\partial x^{2}}+\frac{1}{2} \omega^{2} x^{2}\right] \psi=E \psi
$$

Expression (29) takes the form

$\psi_{\mathrm{A}}^{ \pm}(x)=A \exp \left(\int \pm \sqrt{\omega^{2} x^{2}} \mathrm{~d} x\right)=A \exp \left( \pm \frac{\omega}{2} x^{2}\right)$.
Our ansatz eigenfunction $\psi_{\mathrm{A}}(x)$ can be extracted from (31) enforcing the normalization condition

$$
\begin{gathered}
\psi_{\mathrm{A}}(x) \stackrel{x \rightarrow \pm \infty}{\longrightarrow} 0 \longrightarrow \psi_{\mathrm{A}}(x)=\psi_{\mathrm{A}}^{-}(x) \\
\int \psi_{\mathrm{A}}^{2}(x) \mathrm{d} x=1 \longrightarrow A=\left(\frac{\omega}{\pi}\right)^{1 / 4}
\end{gathered}
$$

and the ansatz grown state eigenfunction adopts the appearance

$$
\psi_{\mathrm{A}}(x)=\left(\frac{\omega}{\pi}\right)^{1 / 4} \exp \left(-\frac{\omega}{2} x^{2}\right)
$$

which coincides with the exact result.

\subsection{Quartic anharmonic oscillator}

The SE for a particle of unit mass in a quartic anharmonic potential reads

$$
\left[-\frac{1}{2} \frac{\partial^{2}}{\partial x^{2}}+\frac{1}{2} \kappa x^{2}+\frac{1}{2} \lambda x^{4}\right] \psi=E \psi
$$

where $\lambda$ is the anharmonicity constant. For the case in which

$$
\kappa=\omega^{2}>0, \quad \lambda>0
$$

expression (29) takes the form

$$
\psi_{\mathrm{A}}^{ \pm}(x)=A \exp \left( \pm \int \sqrt{\omega^{2} x^{2}+2 \lambda x^{4}} \mathrm{~d} x\right)
$$

and, from an elemental integration, one finds that

$$
\psi_{\mathrm{A}}^{ \pm}(x)=A \exp \left[ \pm \frac{1}{6 \lambda}\left(\omega^{2}+2 \lambda x^{2}\right)^{3 / 2}\right] \text {. }
$$

We need to choose $\psi_{\mathrm{A}}(x)=\psi_{\mathrm{A}}^{-}(x)$ in (36), so as to enforce the condition $\psi_{\mathrm{A}}(x) \stackrel{x \rightarrow \pm \infty}{\longrightarrow} 0$. Thus, we have

$$
\psi_{\mathrm{A}}(x)=A \exp \left[-\frac{1}{6 \lambda}\left(\omega^{2}+2 \lambda x^{2}\right)^{3 / 2}\right] \text {. }
$$

We now write $A=N e^{C}$. The real constant $C$ is obtained by the requirement that $\psi$ remain finite in the limit in which $\lambda \rightarrow 0$. The desired eigenfunction takes the form

$$
\psi_{\mathrm{A}}(x)=N \exp \left\{\frac{\omega^{3}}{6 \lambda}\left[1-\left(1+\frac{2 \lambda}{\omega^{2}} x^{2}\right)^{3 / 2}\right]\right\}
$$

where $N$ is determined by enforcing the normalization condition.

Below, the reader may pass judgement on the accuracy of our ansatz via a comparison of our results with other exact or approximate ones and from a plot of the exact GS 
(a)

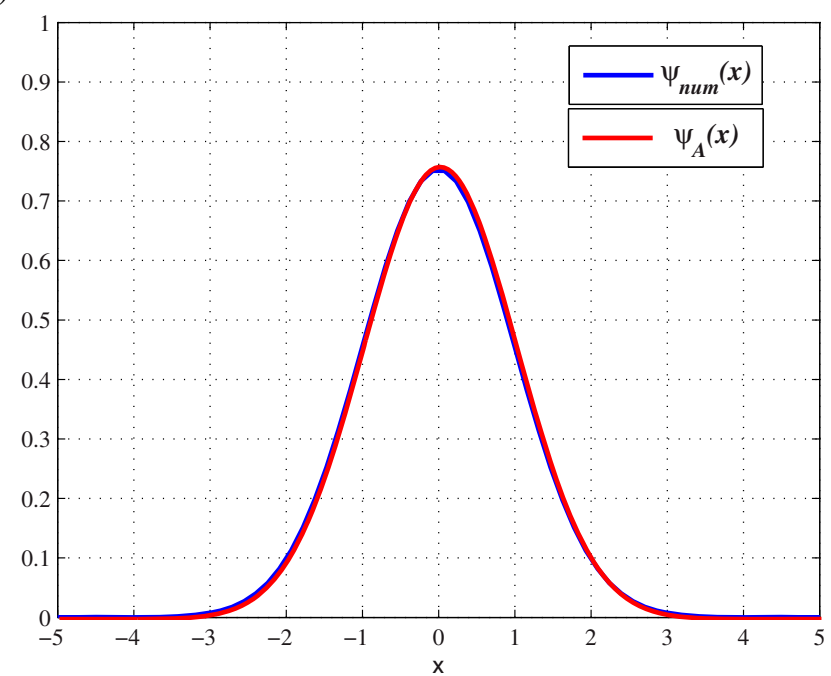

(c)

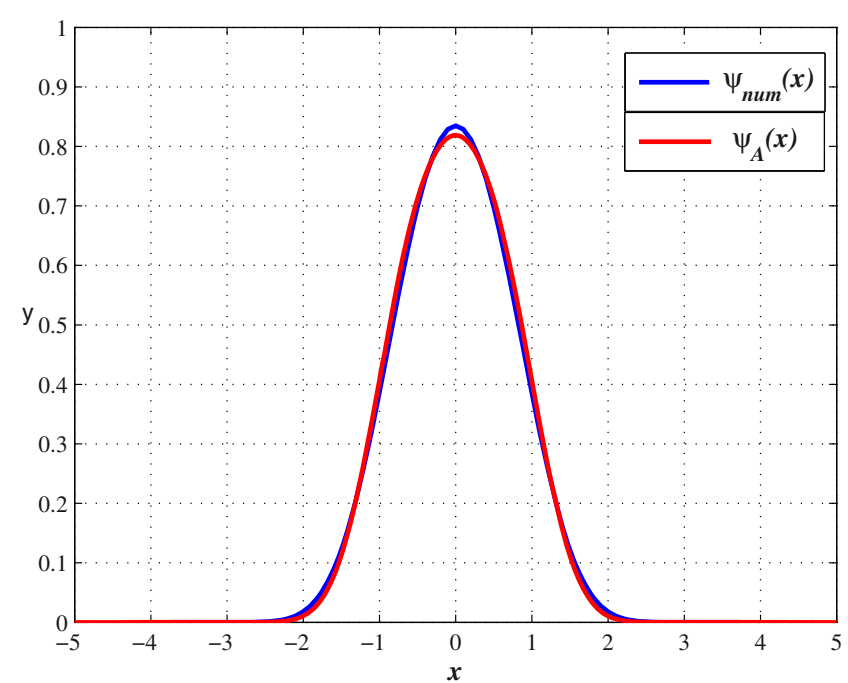

(b)

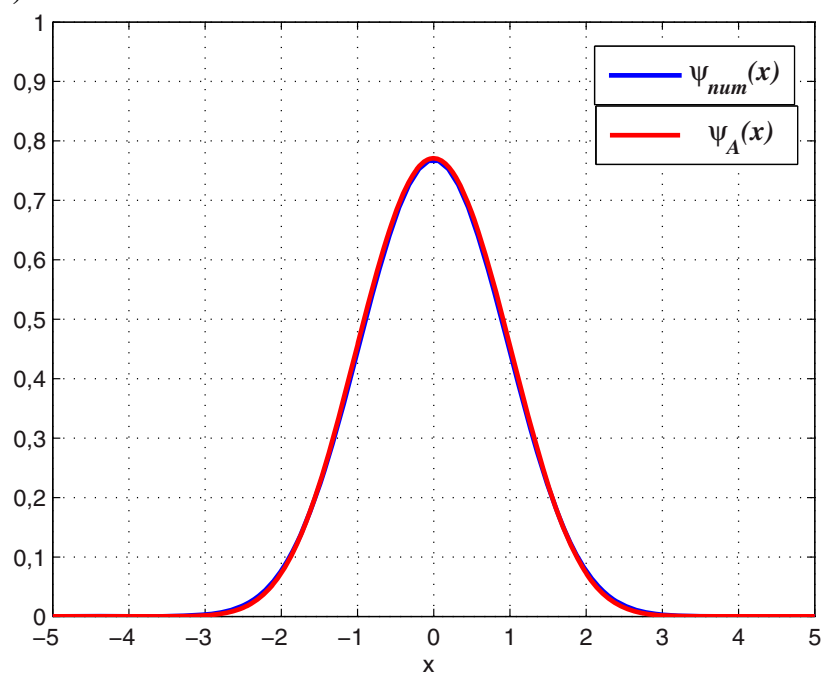

(d)

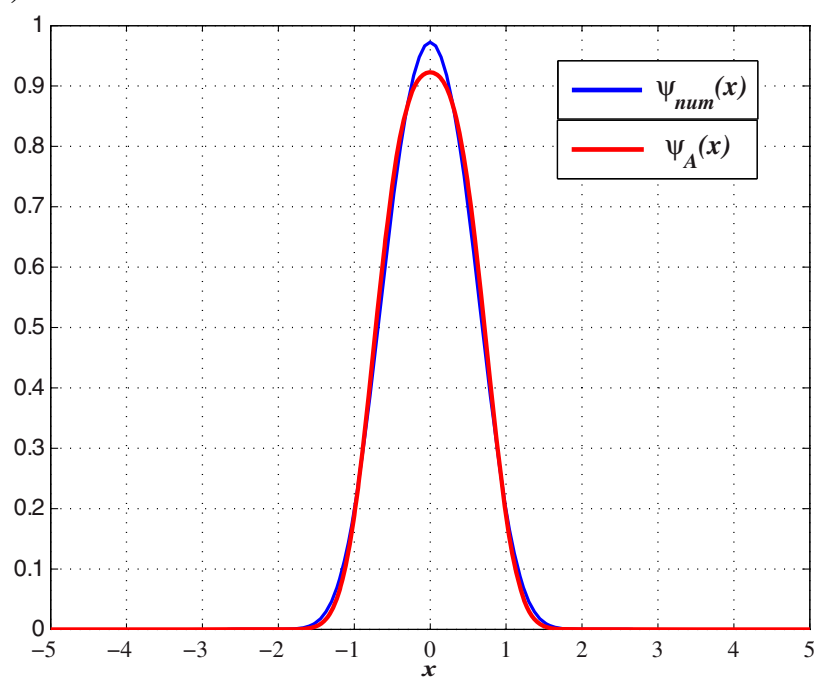

Figure 1. (a) Ground state eigenfunction for $\omega=1$ and $\lambda=0.01$. (b) Ground state eigenfunction for $\omega=1$ and $\lambda=0.1$. (c) Ground state eigenfunction for $\omega=1$ and $\lambda=1$. (d) Ground state eigenfunction for $\omega=1$ and $\lambda=10$.

eigenfunction versus our ansatz one. Finally, we evaluate the approximate eigenenergies for several $\lambda$-values.

- In the limit in which the anharmonicity constant vanishes, one re-obtains the Gaussian form

$$
\lim _{\lambda \rightarrow 0} \psi_{\mathrm{A}}(x)=N_{\text {но }} \exp \left(-\frac{\omega}{2} x^{2}\right),
$$

and when $\omega \rightarrow 0$, the anharmonic oscillator ansatz eigenfunction is given by

$$
\lim _{\omega \rightarrow 0} \psi_{\mathrm{A}}(x)=N_{\mathrm{PAO}} \exp \left(-\frac{\sqrt{2 \lambda}}{3}|x|^{3}\right) .
$$

- The asymptotic behavior is governed by the anharmonicity constant,

for $|x| \gg 1: \quad \psi_{\mathrm{A}}(x) \simeq N \exp \left(-\frac{\sqrt{2 \lambda}}{3}|x|^{3}\right)$.

Consider now the case when $\lambda$ is small. The zeroth-order Wentzel-Kramers-Brillouin (WKB) solution to the present SE (34), for large $|x|$, becomes [20]

$$
\begin{aligned}
\psi_{\mathrm{WKB}}(x)= & C\left(x^{2}+\frac{\lambda}{\omega^{2}} x^{4}\right)^{-1 / 4} \\
& \times \exp \left\{\frac{\omega^{3}}{3 \lambda}\left[1-\left(1+\frac{\lambda}{\omega^{2}} x^{2}\right)^{3 / 2}\right]\right\},
\end{aligned}
$$

where $C$ is a constant. Therefore, in the asymptotic region, $\psi_{\mathrm{WKB}}$ exhibits a rather similar exponential behavior to our ansatz eigenfunction,

$$
\text { for }|x| \gg 1: \quad \psi_{\text {шКв }}(x) \simeq C|x|^{-1} \exp \left(-\frac{\sqrt{\lambda}}{3}|x|^{3}\right) .
$$

- For not too large values of $\lambda$, our ansatz is in good agreement with numerical approximations found in the literature. In each of figures 1(a)-(d), we plotted both the GS numerical-eigenfunctions (using the program 
Table 1. SE GS eigenfunctions evaluated at $x=0$ for $\omega=1$ and several $\lambda$-values. The second column corresponds to the values found in the literature, obtained via a numerical approach to the SE. Our ansatz eigenfunction values are listed in the third column. The relative errors are listed in the fourth column.

\begin{tabular}{lccc}
\hline$\lambda$ & $\psi_{\text {num }}(0)$ & $\psi_{\mathrm{A}}(0)$ & $\epsilon \%)$ \\
\hline 0.01 & 0.7532 & 0.7525 & 0.09 \\
0.1 & 0.7665 & 0.7626 & 0.51 \\
1. & 0.8347 & 0.8104 & 2.91 \\
10. & 0.9714 & 0.9227 & 5.13 \\
\hline
\end{tabular}

Matslise) and the ansatz eigenfunction, for $\omega=1$ and several $\lambda$-values. We display in table 1 some values that the above-mentioned eigenfunctions attain at $x=0$.

- Once we have at our disposal the ansatz GS eigenfunction, we obtain the corresponding eigenvalues by following one of the two procedures indicated below.

The Schrödinger procedure:

$$
\begin{aligned}
E \approx\langle & \left\langle\psi_{\mathrm{A}}|H| \psi_{\mathrm{A}}\right\rangle=\int \mathrm{d} x \psi_{\mathrm{A}}(x) \\
& \times\left[-\frac{1}{2} \frac{\partial^{2}}{\partial x^{2}}+\frac{1}{2} \omega^{2} x^{2}+\frac{1}{2} \lambda x^{4}\right] \psi_{\mathrm{A}}(x) \\
= & \int \mathrm{d} x \psi_{\mathrm{A}}(x)\left[\frac{\omega}{2}\left(1+\frac{2 \lambda}{\omega^{2}} x^{2}\right)^{1 / 2}\right. \\
& \left.+\frac{\lambda}{\omega} x^{2}\left(1+\frac{2 \lambda}{\omega^{2}} x^{2}\right)^{-1 / 2}-\frac{\lambda}{2} x^{4}\right] \psi_{\mathrm{A}}(x) .
\end{aligned}
$$

The Fisher procedure:

From (6) and (12), with $\lambda_{2}=-4 \omega^{2}, \lambda_{4}=-4 \lambda$, we have

$$
\begin{aligned}
\alpha & =I-\sum_{k=1}^{M} \lambda_{k}\left\langle x^{k}\right\rangle=-\sum_{k=1}^{M}\left(\frac{k}{2}+1\right) \lambda_{k}\left\langle x^{k}\right\rangle \\
& =8 \omega^{2}\left\langle x^{2}\right\rangle+12 \lambda\left\langle x^{4}\right\rangle,
\end{aligned}
$$

Evaluating the $x$-moments with the ansatz wave function, we have

$$
\left\langle x^{p}\right\rangle_{\mathrm{A}} \approx \int \mathrm{d} x x^{p} f_{\mathrm{A}}(x)=\int \mathrm{d} x x^{p} \psi_{\mathrm{A}}^{2}(x)
$$

and, accordingly,

$$
E=\frac{\alpha}{8} \approx \omega^{2}\left\langle x^{2}\right\rangle_{\mathrm{A}}+\frac{3}{2} \lambda\left\langle x^{4}\right\rangle_{\mathrm{A}}
$$

Note that we determine $E$ without passing first through an $S E$, which is a nice feature of the present approach. The question of the suitability of our ansatz is answered by looking at table 2 .

\section{Conclusions}

The link between the SE and the Fisher measure has been employed in order to infer, via the pertinent RR, a parameter-free GS ansatz wave function for even, convex
Table 2. SE GS eigenvalues (34) for $\omega=1$ and several values of the anharmonicity constant $\lambda$. The values of the second column correspond to those one finds in the literature, obtained via a numerical approach to the SE. These results, in turn, are nicely reproduced by some interesting theoretical approaches that, however, need to introduce and adjust some empirical constants [21]. Our ansatz values, in the third column, are obtained instead via a parameter-free procedure. The fourth column displays the associated Cramér-Rao bound, which is almost saturated in all instances.

\begin{tabular}{lccc}
\hline$\lambda$ & $E_{\text {num }}$ & $E$ & $I\left\langle x^{2}\right\rangle$ \\
\hline 0.0001 & 0.50003749 & 0.50003749 & 1.000000015 \\
0.001 & 0.50037435 & 0.50037444 & 1.000001477 \\
0.01 & 0.50368684 & 0.50369509 & 1.000129847 \\
0.1 & 0.53264275 & 0.53305374 & 1.000129847 \\
1 & 0.69617582 & 0.70188134 & 1.046344179 \\
10 & 1.22458704 & 1.25080186 & 1.099588057 \\
100 & 2.49970877 & 2.57093830 & 1.123451126 \\
1000 & 5.31989436 & 5.48276171 & 1.130099216 \\
\hline
\end{tabular}

potentials, a rather large family indeed. Its parameter-free character notwithstanding, our ansatz provides good results, as evidenced by the examples examined here. We believe that, for an even, convex potential function, excellent results could eventually be achieved if perturbative expansion methods were developed using this ansatz function as the zeroth-order approximation.

Finally, we emphasize that our procedure incorporates only the knowledge of the virial theorem, via the Legendre symmetry that underlies the connection between Fisher's measure and the SE. One may again speak here of the power of using symmetry considerations in devising physical treatments.

\section{Acknowledgments}

This work was partially supported by project 11/X301 of the Universidad Nacional de La Plata, Argentina and by projects FQM-2445 and FQM-207 of the Junta de Andalucia (Spain). AP acknowledges support from the senior grant CEI Bio-Tic GENIL-SPR. This work was partially supported by the following grants: (i) project PIP1177 of CONICET (Argentina) and (ii) project FIS2008-00781/FIS (MICINN) and FEDER (EU) (Spain).

\section{References}

[1] Fernandez F M and Castro E A 1987 Hypervirial Theorems (Berlin: Springer)

[2] Plastino A R, Casas M, Plastino A and Puente A 1995 Phys. Rev. A 522601

[3] Frieden B R, Plastino A, Plastino A R and Soffer B H 1999 Phys. Rev. E 6048

[4] Reginatto M 1998 Phys. Rev. A 581775

[5] Flego S P, Frieden B R, Plastino A, Plastino A R and Soffer B H 2003 Phys. Rev. E 68016105

[6] Flego S P, Plastino A and Plastino A R 2011 Ann. Phys. 3262533

[7] Flego S P, Plastino A and Plastino A R 2011 Physica A 3902276

[8] Flego S P, Plastino A and Plastino A R 2011 Physica A 3904702

[9] Flego S P, Plastino A and Plastino A R 2011 J. Math. Phys. $\mathbf{5 2} 082103$ 
[10] Brillouin L 1956 Science and Information Theory (New York: Academic)

[11] Katz A 1967 Principles of Statistical Mechanics (San Francisco, CA: Freeman)

[12] Plastino A R and Plastino A 1993 Phys. Lett. A 181446 Casas M, Garcias F, Plastino A and Serra L I 1995 Physica A 213376

[13] Frieden B R 2004 Science from Fisher Information: A Unification (Cambridge: Cambridge University Press)

[14] Luo S 2000 Lett. Math. Phys. 53243

[15] Flego S P, Plastino A and Plastino A R 2011 J. Mod. Phys. 21390
[16] Feynman R P 1939 Phys. Rev. 56340

[17] Desloge A 1968 Thermal Physics (New York: Holt, Rinehart and Winston)

[18] Greiner W and Müller B 1988 Quantum Mechanics. An Introduction (Berlin: Springer)

[19] Biosca A F 2001 Lecture Notes in Economics and Mathematical Systems vol 502 (Berlin: Springer) pp 189-217

[20] Bender C M and Wu T T 1969 Phys. Rev. 1841231

[21] Banerjee K, Bhatnagar S P, Choudhry V and Kanwall S S 1978 Proc. R. Soc. Lond. A 360575

Banerjee K 1978 Proc. R. Soc. Lond. A 364265 